\title{
Markov Chain Combination Prediction Model and Its Application in Stock Market
}

\author{
Qingxin Zhou \\ School of Basic Science, Harbin University of Commerce, Harbin 150028, China \\ E-mail: zhouqingxin1981@126.com
}

Keywords: Markov Chain Prediction Method; The Median Method; Level Characteristic Value Method; Combination Model; The Stock Market

\begin{abstract}
Markov process is a very important means to describe dynamic stochastic phenomena. First, Markov chain median value prediction method and Markov chain level Characteristic value method are introduced. Based on these predictions, the thought and steps of the combined prediction model are given. Secondly, in the part of empirical research, this paper uses Markov chain combined prediction model to predict the stock price of China railway in China's stock market and obtain the predicted value of the combination. Compared the predicted value with the predicted value obtained by other single methods, the combined predicted value is more accurate., thus it is concluded that combined forecasting method is superior to other Markov prediction method, and enrich the theory of Markov chain prediction system, it also manifests the combination of prediction and Markov chain prediction method has a strong suitability.
\end{abstract}

\section{Introduction}

Because a single Markov chain prediction model only represents the laws of data from one aspect, a large amount of effective information implied in the data is lost ${ }^{[1]}$. The Markov chain combination prediction theory proposed in this paper is to combine the advantages of other kinds of prediction models for comprehensive prediction., namely through the level characteristic value to various Markov chain prediction method of state predictive value converted to a specific value, and with the method of combination forecast to predict, get a better prediction results. Markov chain combination prediction method integrates other prediction methods and collects effective information of each single prediction model, so the combination prediction improves the accuracy and accuracy of the prediction results. ${ }^{[2]}$

\section{Preliminary Knowledge}

Generally, the prediction results obtained by some Markov chain prediction methods are an interval. The median method is usually used to convert the prediction interval into a specific value. That is, if the prediction interval obtained by Markov chain prediction method is $\left[Q_{1}, Q_{2}\right]$, then the predicted value is taken as the median value $\frac{Q_{1}+Q_{2}}{2}$ between the regions. This method is called Markov chain median prediction method ${ }^{[3]}$.

\subsection{Markov Chain Level Characteristic Value Prediction Model}

If the method of level Characteristic value in fuzzy set theory is adopted, the state can be transformed into a specific value through the combination formula, and the accuracy of the predicted value will be higher than the median value of the interval. Combining this level Characteristic value method with Markov chain prediction method, the resulting prediction model is called Markov chain level Characteristic value prediction model ${ }^{[4]}$.

The specific steps of establishing the Markov chain level Characteristic value prediction model are 
as follows:

First, by Markov chain prediction method, the state interval of the future process is determined according to the principle of "maximum probability", and the prediction interval is set as state $i_{0}$.

Secondly, the following indicators are determined: $A_{i_{0}}, B_{i_{0}}$ are the upper and lower limits corresponding to the prediction interval $i_{0}, J_{i_{0}}=\sum_{k=1}^{n} k d_{k}$ is the rank characteristic value, $d_{k}$ is the weight of the $k$ th state, $d_{k}=\frac{p_{k}{ }^{2}}{\sum_{k=1}^{n} p_{k}{ }^{2}}, k=1,2, \cdots n$. and $p_{i_{0}}$ are the probability corresponding to the state $i_{0}$, namely the "maximum probability".

Finally, if (1) $J>i$, the predicted value is $\frac{A_{i} J_{i}}{i+0.5}$.

If (2) $J \leq i$, the predicted value is $\frac{B_{i} J_{i}}{i-0.5}$.

In conclusion, the prediction interval state is transformed into a specific value by this model.

\subsection{Markov Chain Combined Prediction Model}

The Markov chain combined prediction model established in this paper integrates other forecasting methods. The specific process is as follows:

First of all, for the same prediction problem, the hypothesis can build $N$ different prediction model.

Secondly, the predicted values of each model at time $t$ are calculated respectively. If $b_{t}$ is the real value at time $t, f_{i t}$ is the predicted value at time $t$ obtained by the $i$ th model, then $E_{i t}$ is the prediction error at time $t$ caused by the $i$ th model, where $t=1,2, \cdots, n$.

Finally, if

$$
f_{t}=\sum_{i=1}^{N} k_{i} f_{i t},
$$

Where $k_{i}$ is the weight of the $i$ th model in the composite model, Then $f_{t}$ represents the combined prediction value of the ith model, and $E_{t}=b_{t}-f_{t}=\sum_{i=1}^{N} k_{i} E_{i t}$ represents the prediction error of the combined model of the ith model at time t. Suppose $e=\left(e_{1}, e_{2} \cdots, e_{n}\right)$, where $e_{i}$ is the error vector of the ith model, and $e=E^{T} E$ is the prediction error matrix, then there are $e=E^{T} E$, and $K=\left(k_{1}, k_{2}, \cdots, k_{N}\right)$ is the optimal weighted coefficient vector, Satisfying conditions

$$
K=\frac{e^{-1} R}{R^{T} e^{-1} R} \text { and } R=[1,1, \cdots 1]^{T} .
$$

Formula (1) is the combined prediction model. In particular, if the $N$ prediction models constructed are based on Markov chain, formula (1) is called Markov chain based composite prediction model. Formula (2) is the optimal weighted coefficient vector ${ }^{[5-7]}$.

In addition, with the addition and improvement of various Markov prediction models, the accuracy of the combined prediction model will be gradually improved.

\section{The Empirical Analysis}

With the improvement of people's living standard, people are more and more willing to invest in high-risk and high-return investment projects, and the stock market is more and more active. But because stock prices have a certain degree of volatility, if we invest in stocks blindly, it may not really 
bring us benefits.

This chapter will use combination prediction theory based Markov chain to predict stock prices. Taking the closing price of China railway from October 1, 2015 to March 27, 2016 as a sample, the closing price of this stock is predicted to be on March 28, 2016. (data source: RESSET database)

Analysis of the China railway shares in October 2015 - March 2016 record closing price time series data found that the China railway stock's closing price along with the time of there are many complex and uncertainty in the process of composition, data fluctuation is bigger, also closing sequence has the Markov property.

According to the above analysis, the composite forecast method is used to predict the closing price of China railway stock. By comparing the combined predictive value with the median and level values obtained by Markov chain prediction method, it is concluded that the combined prediction model results are closer to the actual results. In conclusion, the portfolio prediction model is of great importance, and it is of guiding significance to investors how to invest in stocks not only in China railway stocks.

\subsection{Markov chain median value prediction method and level Characteristic value prediction method}

Firstly, based on historical data, a specific prediction interval is obtained by Markov chain prediction method. Since the closing price of a stock fluctuates all the time, it can be regarded as unchanged when it fluctuates within a certain range, so in these data, plus or minus 0.5 is the allowed range. If the increase is greater than 0.5 , it is denoted as state 1 . The fluctuation range is denoted as state 2 and 2 as flat state in $\left[\begin{array}{ll}-0.5 & 0.5\end{array}\right]$. A decrease of more than 0.5 is denoted as state 3 and State 3 is the descending state, as shown in Table 1.

Table 1. State Division Table of Stocks Closing Prices of China Rail Way

\begin{tabular}{ccc}
\hline State & Grades & Standards for Grading \\
\hline 1 & Rise & $0.5 \leq x$ \\
2 & Flat & $-0.5 \leq x<0.5$ \\
3 & Fall & $x<-0.5$ \\
\hline
\end{tabular}

Table 1 gives the Standards for Grading. Statistics are as follows: the closing price of 42 days is in an upward state compared to the previous day, 26 days is in a flat state, and 48 days is in a downward state. The last day was 2, or "flat," and there was no state transfer data, so the total number of flat was 25 . The number of times from the rising state to the rising state is 16 , the frequency is $\frac{16}{42}$, And so on. The one-step transition probability matrix of the Markov chain is as follows:

$$
p=\left[\begin{array}{lll}
0.3810 & 0.1667 & 0.4523 \\
0.1600 & 0.2800 & 0.5600 \\
0.4583 & 0.2083 & 0.3334
\end{array}\right]
$$

Because the 116th day (March 7) in the flat state, so the initial state vector is $S^{(0)}=\left(\begin{array}{lll}0 & 1 & 0\end{array}\right)$, using it to predict the 117th day (March 8) probability of closing price in the each state, the 117th day state probability vector as follows:

$$
S^{(1)}=S^{(0)} p=\left(\begin{array}{lll}
0 & 1 & 0
\end{array}\right)\left[\begin{array}{lll}
0.3810 & 0.1667 & 0.4523 \\
0.1600 & 0.2800 & 0.5600 \\
0.4583 & 0.2083 & 0.3334
\end{array}\right]=\left(\begin{array}{lll}
0.1600 & 0.2800 & 0.5600
\end{array}\right)
$$

According to the "maximum probability principle", the stock price on March 8 can be predicted to be in a state of "decline". In the real stock market, the closing price on March 8 is 8.05, which is lower than the previous day. Compared with the previous day, it was in a state of decline, and the predicted 
results were consistent with the real situation.

The median method and the level Characteristic value method are respectively used to calculate the data obtained by Markov chain prediction method, and then the prediction value of March 28 is obtained. The predicted results are 10.21 and 7.27 respectively.

\subsection{Markov Chain Combined Prediction Model}

First of all, through the formul (2), two model weight coefficient were 0.2653 and 0.7347 . The predicted value obtained by the median method and the level Characteristic value method was put into the combined prediction model (1), and the predicted result of the closing price of March 28, 2016 was 8.049982, as shown in Table 2.

Table 2 Comparison Table of Predicted Values of the Three Models

\begin{tabular}{lcc}
\hline \multicolumn{1}{c}{ Prediction method } & Predictive value & Error \\
\hline Markov chain median prediction method & 10.21 & 2.16 \\
Markov chain level Characteristic value method & 7.27 & 0.78 \\
Markov chain combined prediction model & 8.049982 & 0.000018 \\
\hline
\end{tabular}

It is not hard to see from the Table 2 combination forecast based on the Markov chain method is not only the most accurate, and it is the most realistic value, the level characteristic value forecast method compared with interval median forecast method, the accuracy of the prediction has made great progress, and the error of the prediction relative decline, one of the biggest error was in the method, the minimum error is combination forecast. Through the combination forecast method of Markov chain prediction of stock price is relatively accurate, shareholders can refer to this number to make reasonable investment, for many people to avoid unnecessary risks.

\section{Conclusion}

Markov chain combined prediction model is suitable for the prediction of data with abundant data, large fluctuation range and large influence components. Under certain conditions, Markov chain combination forecasting model has unique advantages. In the application of combination forecast method of Markov chain, we will find that this method not only calculation is more convenient, and compared with other methods, its accuracy is higher, so Markov chain combination forecast method has a certain practical significance and application value.

\section{References}

[1] Xueqiang Ye, Yufeng Gui. Statistics and analysis Decisions, 2018 (02): 69-72. In Chinese

[2] Huidian Long, Guangle Yan. Mathematical statistics and management, 2017, 36 (02):200-207. In Chinese

[3] Chao Zhang, Fengting Jia. Journal of Liaoning university of engineering and technology, 2011, 30 (6): 963-966. In Chinese

[4] Minghui Jiang, Yi Cao. Statistics and decision-making, 2006(23): 43-44. In Chinese

[5] Jun Wang, Juan Wang. Stochastic Process and its Application in the Financial Field [M]. Beijing: Tsinghua University, press; Beijing university press.2009, 46-66. In Chinese

[6] Chao Zhang. Combined Prediction Model Based on Markov Chain [D]. Liaoning University of engineering and technology, 2012. In Chinese

[7] liang Zhang, Shijuan Guo. Hebei engineering technology Journal of college, March 2014:18-21. In Chinese 